\title{
Hutchinson (1828-1913), su historia, su tríada y otras tríadas de la medicina
}

\author{
PABLO YOUNG, BÁRBARA C. FINN, \\ DÉBORA PELLEGRINI, JULIO E. BRUETMAN

\section{Hutchinson and his history}

The prolific life of Jonathan Hutchinson is reviewed. The importance of triads, a set of signs and symptoms that lead to the suspicion of the presence of one or more clinical entities, that are a demonstration of associative thinking, is also discussed. Nowadays, when technology plays a very important role in medicine, we would like to underscore the value of history taking and physical examination as useful clinical tools.

(Rev Med Chile 2010; 138: 383-387).

Key words: History of Medicine; Medical history taking; Physical examination.

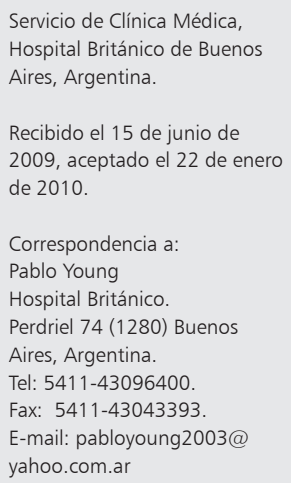

\section{Perspectiva histórica}

Sir Jonathan Hutchinson (Figura 1) nació en Selby, Yorkshire, Inglaterra, el 23 de julio de $1828^{1}$. Durante sus estudios iniciales de medicina en la Small York School, quedó impactado por las enseñanzas de Thomas Laycock, quien más tarde fuera profesor en Edinburgo. En 1850 se trasladó a Londres para graduarse en la Escuela de Medicina del St. Bartholomew's Hospital, y al poco tiempo fue el médico más consultado de Londres, dado su amplio conocimiento en todos los campos de la medicina ${ }^{2}$. En esta escuela, Hutchinson fue influenciado y ayudado por su mentor, Sir James Paget (1814-1899), quien lo acompañó en la evaluación de los pacientes ambulatorios e internados. Al igual que Paget, Jonathan Hutchinson fue nombrado Caballero del Imperio Británico en $1908^{3}$. Era tan grande el respeto que Paget le tenía por la habilidad con que resolvía aun los casos más complicados, que habitualmente le enviaba pacientes para una segunda opinión ${ }^{3,4}$. Fue una personalidad médica reconocida internacionalmente. Promovido por McWhinnie, trabajó como dermatólogo en el Blackfriars Hospital. Por otro lado, se desempeñó como oftalmólogo en el Royal London Ophthalmic Hospital y como especialista

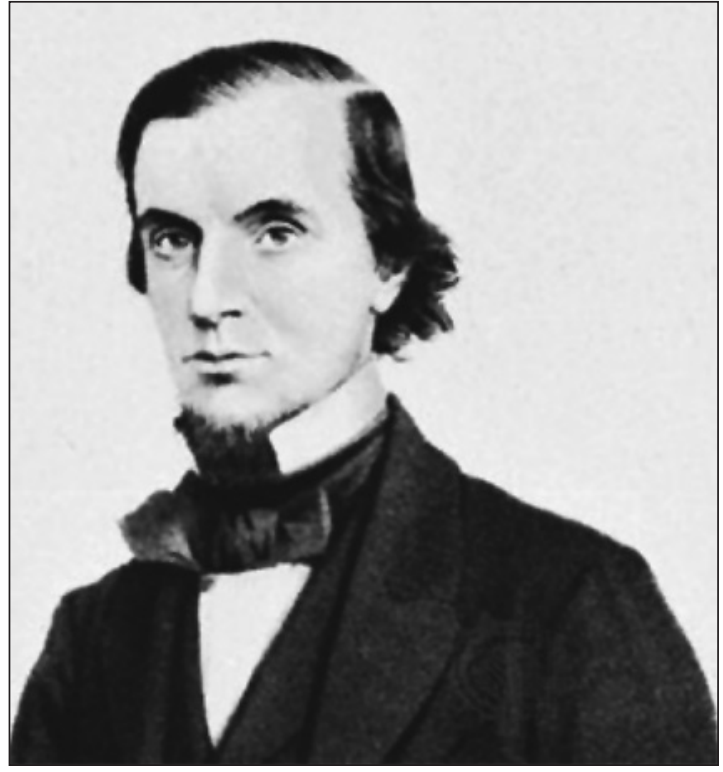

Figura 1. Jonathan Hutchinson.

en enfermedades infecciosas en el Lock Hospital. Desarrolló su labor de clínico en el London Chest Hospital y cirujano general (esta vez alentado por Paget) en el London and Metropolitan Hospitals. Fue presidente de la Hunterian Society en 1869 y 


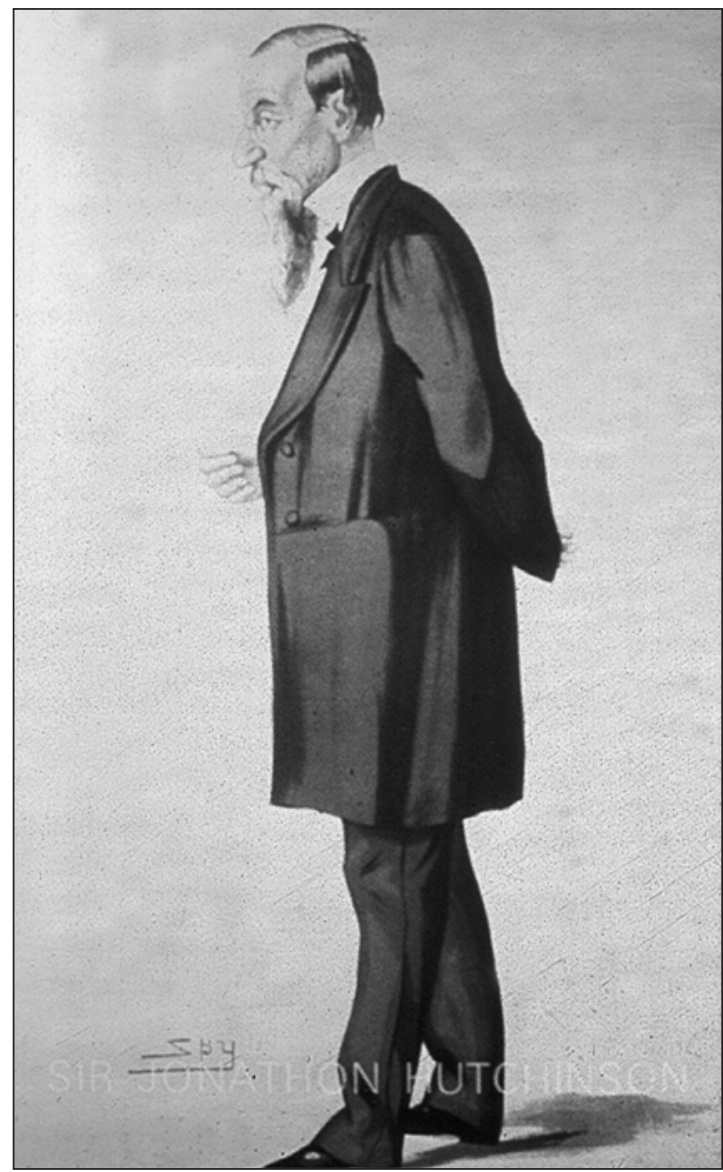

Figura 2. Jonathan Hutchinson.

1870, de la Pathological Society of London en 1879, de la Ophthalmological Society of the United Kingdom en 1883, de la Neurological Society en 1887, del Royal College of Surgeons en 1889, de la Medical Society of London en 1892, de la Royal Medical and Surgical Society entre 1894 y 1896 y presidente del Congreso Internacional de Dermatología en $1896^{1-4}$. También fue editor en jefe del British Medical Journal (BMJ) y entre los años 1890 y 1900 escribió artículos que aparecieron trimestralmente en el Archives of Surgery. Publicó más de 1.200 artículos médicos ${ }^{3}$.

De personalidad oscura, indiferente, cerrada, sin humor, apegado a las tradiciones religiosas (miembro de una familia de cuáqueros). Tenía una figura longilínea y barba larga, aun cuando en esos tiempos ésta ya no se usaba (Figura 2). Gran observador y recolector de datos, fue uno de los clínicos más brillantes de su época, excelente profesor y maestro de la medicina ${ }^{4}$. Según W.B. Bean, de existir un Premio Nobel para Maestros, Jonathan Hutchinson lo hubiera merecido 5 .

En enero de 1869, un paciente de 58 años, el señor John W. Mortimer, visitó al Dr. Hutchinson en el Blackfriars Hospital, por unas placas purpúreas de crecimiento gradual desde los dos años previos, que se ubicaban simétricamente en los muslos y las manos. Estas lesiones eran indoloras y nunca se ulceraban, a diferencia del lupus vulgar, por lo que en un principio Hutchinson le dio a esta entidad el nombre del paciente llamándola "enfermedad de Mortimer". En 1877 Hutchinson publicó el caso bajo el título "Case of livid papillary psoriasis", siendo la primera descripción de sarcoidosis (del griego: condición similar a la carne) con afectación cutánea, hoy conocida como enfermedad de Besnier-Boeck-Shaumann.

Además de la sarcoidosis (Tabla 1), en 1858 describió la tríada de la sífilis congénita que lleva su nombre, que consta de queratitis intersticial, sordera y "dientes de Hutchinson".

Describió la pupila de Hutchinson-Boeck en 1865 (la pupila del lado de la lesión está midriática y fija debido a una lesión del III par, mientras que la contralateral se contrae al estimulo lumínico) y la progeria (envejecimiento precoz o síndrome de Hutchinson-Gilford) en 1886. En 1896 reportó 2 hermanas con máculas pigmentadas en los labios y mucosa yugal, entidad que después se llamó síndrome de Hutchinson-Weber-Peutz y cuando las lesiones pigmentadas se asocian a pólipos hamartomatosos del intestino delgado constituyen el síndrome de Peutz-KlostermannTouraine-Jeghers ${ }^{1-4}$.

Jonathan Hutchinson fue el primero en describir la arteritis de la temporal, en 1889, hoy llamada enfermedad de Hutchinson-HortonGilmour-Magath-Brown. Además describió la balanitis atrófica, el lentigo maligno, el eczema dishidrótico, la degeneración macular de coroides o enfermedad de Hutchinson-Tay (degeneración senil de coroides con pérdida visual progresiva, con manchas amarillentas alrededor de la mácula). A nivel corneal describió un área de neovascularización de color salmonado que observó en la queratitis sifilítica la cual se conoce como parche de Hutchinson².

Describió en la oftalmoplejía externa progresiva o enfermedad de Von Gräfe-Fuchs, la 
Tabla 1. Cronología de las descripciones de Jonathan Hutchinson

\begin{tabular}{|c|c|}
\hline Año & $\begin{array}{l}\text { Descripción de enfermedad, } \\
\text { síndrome, signo o procedimiento }\end{array}$ \\
\hline 1858 & Tríada de la sífilis congénita \\
\hline 1865 & Pupila de Hutchinson-Boeck \\
\hline 1865 & $\begin{array}{l}\text { Descripción de lo que hoy se conoce como } \\
\text { síndrome de Claude Bernard-Horner }\end{array}$ \\
\hline 1874 & Primera cirugía de intususcepción \\
\hline 1877 & Hutchinson describe la sarcoidosis \\
\hline 1878 & Prurigo de verano \\
\hline 1882 & Varicela gangrenosa \\
\hline 1883 & $\begin{array}{l}\text { El edema sólido de la cara después de la } \\
\text { erisipela }\end{array}$ \\
\hline 1886 & La progeria \\
\hline 1889 & La hiperqueratosis por arsénico \\
\hline 1889 & Arteritis de la temporal \\
\hline 1889 & Balanitis atrófica \\
\hline 1889 & El lentigo maligno \\
\hline 1889 & El eczema dishidrótico \\
\hline 1889 & La degeneración macular de coroides \\
\hline 1889 & Parche de Hutchinson \\
\hline 1889 & $\begin{array}{l}\text { Papa de Hutchinson: tumor del cuerpo } \\
\text { carotídeo }\end{array}$ \\
\hline 1889 & Facie de Hutchinson \\
\hline 1889 & $\begin{array}{l}\text { La melanosis de Hutchinson o síndrome de } \\
\text { Dubruilh-Chambardel }\end{array}$ \\
\hline 1889 & Máscara de Hutchinson \\
\hline 1889 & Tumor melanótico del lecho ungueal \\
\hline 1890 & $\begin{array}{l}\text { Los nódulos en jalea de manzana del lupus } \\
\text { vulgar }\end{array}$ \\
\hline 1890 & El angioma serpinginoso \\
\hline 1892 & Reconoció la morfea y la esclerodermia \\
\hline 1896 & $\begin{array}{l}\text { Máculas pigmentadas en los labios y mucosa } \\
\text { yugal }\end{array}$ \\
\hline 1896 & $\begin{array}{l}\text { Acroesclerodermia: reconoció la asociación } \\
\text { entre el fenómeno de Raynaud y la esclero- } \\
\text { dermia }\end{array}$ \\
\hline 1897 & $\begin{array}{l}\text { Neuroblastoma con metástasis craneales o } \\
\text { en órbita }\end{array}$ \\
\hline 1897 & Ulceración del eritema indurado de Bazin \\
\hline 1899 & $\begin{array}{l}\text { El queratoacantoma (úlcera crateriforme de } \\
\text { la cara) }\end{array}$ \\
\hline
\end{tabular}

facies de Hutchinson o mirada de astrónomo o de adorador de la luna. La melanosis de Hutchinson o síndrome de Dubruilh-Chambardel es una condición previa al melanoma que consta de lesiones pequeñas en la piel de la cara, marrón oscuro o sepia ${ }^{2,4}$.

Describió en la neurosífilis la sensación subjetiva de sentir la cara cubierta con algodón (máscara de Hutchinson), y el síndrome melanótico de Hutchinson o síndrome de Hutchinson III (tumor melanótico del lecho ungueal). También se llama síndrome de Hutchinson, cuando el neuroblastoma (tumor de Hutchinson-Abercrombie) presenta metástasis craneales o en órbita y síndrome de Pepper cuando éstas se encuentran en el hígado. Describió lo que se dio a llamar variedad de Hutchinson del eritema indurado de Bazin, que es cuando en su evolución éste se ulcera. Por último, también describió, en 1865, lo que hoy se conoce como síndrome de Claude Bernard-Horner (que fuera descripto por Bernard en 1858 y Horner en 1869, si bien se sabe que lo describieron otros autores previamente, como François Pourfour du Petit y Edward Selleck Hare $)^{1-4}$.

Entre las teorías erróneas sobre la etiopatogenia de las enfermedades, creía que la lepra (enfermedad de Hansen) aparecía por la ingestión de pescado en mal estado y que la urticaria pigmentosa que él describiera junto a Nettleship, la producía la picadura de insectos ${ }^{2}$.

Uno de sus discípulos más conocidos fue Edward Nettleship (1845-1913), quien describió la mastocitosis cutánea o urticaria pigmentosa el 18 de septiembre de 1869 en el British Medical Journal (en este articulo se evidencia una clara influencia de Jonathan Hutchinson) y la enfermedad de Nettleship-Falls o albinismo ocular asociado al X, entre otras descripciones ${ }^{2}$.

En 1872, Hutchinson compró una extensa propiedad en Haslemere, Surrey, a donde se mudó con su esposa y diez hijos. Fundó en esta ciudad la London Polyclinic of Postgraduate School of Medicine y el Museo de Educación de Instrucción Popular en Historia Natural, en $1890^{1,2}$.

Jonathan Hutchinson falleció el 23 junio de 1913 in Haslemere, Surrey, Inglaterra. Sus restos descansan en Cavendish Square W1.

Hutchinson fue uno de los médicos más destacados durante el siglo XIX en Inglaterra, así como un gran observador. 


\section{Las tríadas}

Así como la tríada de Hutchinson es una manifestación cardinal de la sífilis congénita, se pueden encontrar en la medicina otras tríadas. Las tríadas son un conjunto de signos y síntomas que permiten sospechar la existencia de una o varias entidades clínicas, siendo a su vez una demostración del pensamiento asociativo. Se han descripto en la medicina alrededor de doscientas. Además de la tríada de Hutchinson ya mencionada, debemos recordar la tríada de Saint que consta de hernia hiatal, litiasis biliar y diverticulosis colónica ${ }^{7}$. Como se ve, la tríada de Hutchinson se compone por signos de una misma enfermedad mientras que la de Saint es la suma de múltiples entidades, no habiendo en este caso base fisiopatológica para la coexistencia de estas tres enfermedades. Saint fue un cirujano sudafricano que enfatizó la importancia de considerar la posibilidad de múltiples enfermedades separadas en un paciente, cada vez que su historia o su examen fueran atípicos para un único diagnóstico ${ }^{7}$. Saint enfatizó que más de una enfermedad puede ser la causa de los síntomas y signos en un mismo paciente. En el mismo sentido una frase de John Hickam dice: "Un paciente puede tener tantos diagnósticos como se nos dé en gana", a esto se lo ha llamado "sentencia de Hickam". Debido a que los médicos nos enfrentamos a un número creciente de pacientes con una multitud de condiciones agudas y crónicas, las visiones de Saint y de Hickam deben ser consideradas en la práctica de la medicina moderna.

Contrapuesto con la visión previa y defendiendo la teoría del "unicismo", encontramos a William Osler, quién trató de explicar los síntomas y signos de los pacientes con un único diagnóstico? Se debe al sacerdote franciscano Guillermo de Occam la frase "la pluralidad no se debe postular sin necesidad". Este principio ya formaba parte de la filosofía medieval, aunque fue Occam quien lo utilizó de forma filosófica. En su forma más simple, el principio de Occam indica que las explicaciones nunca deben multiplicar las causas sin necesidad ${ }^{10}$. Cuando dos explicaciones se ofrecen para un fenómeno, la explicación completa más simple es preferible como la famosa frase "si escucha galopar piense en caballos y no en cebras". La explicación más simple y suficiente es la más probable -más no necesariamente la verdadera-, según el principio de Occam. A medida que la población envejece y mayor cantidad de personas llegan a edades avanzadas, la probabilidad que éstas presenten simultáneamente dos, tres, o más diagnósticos aumenta.

¿Cómo mantener un equilibrio utilizando la filosofía de Occam o la de Saint en la práctica de la medicina en nuestros tiempos? Tenemos que convivir con ambas, porque ambas son útiles, pero tenemos que acostumbrarnos a no mirar a Saint como sinónimo de facilismo o de mala práctica de la medicina, y a saber que no siempre se puede ser unicista. Esto no significa abandonar el principio de unicismo que se nos ha inculcado desde el comienzo de nuestra formación universitaria, sino que significa tener la mente abierta a otras posibilidades cuando el caso no parece resolverse con una explicación única. La observación crítica, usando tecnología simple, asociando signos y síntomas es la fundación científica del arte en la cama del enfermo.

A través de la historia, los médicos se han dedicado a encontrar pistas que conduzcan a un diagnóstico correcto en el caso de patologías comunes y no comunes. Si bien algunas de estas tríadas pueden ser fruto de la casualidad, la mayoría se deben a un profundo esfuerzo de observación y tiempo. Si prestamos atención, la mayoría de ellas fueron descriptas en el siglo XIX o al comienzo del siglo XX, cuando existía un "tiempo" para pensar, reflexionar, asociar y describir.

En el mundo de la medicina de hoy, en donde la tecnología juega un rol importante, como dice Alberto Agrest ${ }^{11}$ "hemos llegado a escuchar poco, explicar menos, pedir muchos exámenes auxiliares y decidir sobre la base de lo que los exámenes nos informan", perdiendo así el valor relevante de la anamnesis y el examen físico. La semiología, la anamnesis y el contacto físico constituyen las bases no sólo del diagnóstico sino también de la relación médico paciente, el abandono de estos recursos será perder humanidad en la actividad médica, perder el placer de resolver un diagnóstico con una pregunta oportuna, con una auscultación que nadie ha hecho, con una palpación que descubre dolor donde las ecografías, tomografías y resonancia no habían demostrado anormalidad ${ }^{8}$. Quizás no estamos asistiendo al ocaso de la semiología, sino al renacer de una nueva semiología, la que pone el esfuerzo en el arte de descubrir lo que los recursos auxiliares no consiguen hacer o lo hacen con mayor agresividad, con mayor riesgo y siempre con 
mayor costo y diluyendo responsabilidades ${ }^{12}$. Nos parece que el recuerdo de personalidades como la de Hutchinson, con sus descripciones novedosas, son un homenaje que la medicina adeuda.

\section{Referencias}

1. James DG, Sharma OP. From Hutchinson to now: a historical glimpse. Curr Opin Pulm Med 2002; 8: 416-23.

2. Branford WA. Hutchinson and Nettleship, nettlerash and albinism. Br J Dermatol 2000; 143: 16-22.

3. Ellis H. Jonathan Hutchinson (1828-1913). J Med Biog 1993; 1: 11-6.

4. McKusick VA. The Gordon Wilson Lecture: The clinical legacy of Jonathan Hutchinson (1828-1913): syndromology and dysmorphology meet genomics. Trans Am Clin Climatol Assoc 2005; 116: 15-38.

5. Bean WB. Jonathan Hutchinson. Arch Intern Med 1965;
116: 1-3.

6. Hutchinson J. Case of livid papillary psoriasis. Illustrations of Clinical Surgery. London J and A Churchill $1877,42$.

7. Schwartz J. Saint's triad: cholelithiasis, hiatus hernia, and diverticulosis of the colon in the same patient. Am J Gastroenterol 1960; 34: 509-12.

8. Ross JC. John Bamber Hickam. Physician, educator, investigator. Arch Intern Med 1971; 127: 571-3.

9. Bliss M. William Osler at 150. CMAJ 1999; 161: 831-4.

10. Lo Re V 3rd, Bellini LM. William of Occam and Occam's razor. Ann Intern Med 2002; 136: 634-5.

11. Agrest A. Pasado, presente y futuro en la enseñanza de la medicina clínica. En: Cámera MI, Romani A, Madoery C, Farías J, editors. Avances en medicina '94. Buenos Aires: Sociedad Argentina de Medicina; 1994, p 295-306.

12. Agrest A. El ocaso de la semiología. Medicina (Buenos Aires) 2008; 68: 175. 